\title{
Thermodynamic stability of hnRNP A1 low complexity domain revealed by high-pressure NMR
}

\author{
Jeffrey Levengood ${ }^{1}$, Jake Peterson ${ }^{2}$, Blanton Tolbert ${ }^{1}$, and Julien Roche ${ }^{2}$ \\ ${ }^{1}$ Case Western Reserve University \\ ${ }^{2}$ Iowa State University
}

September 1, 2020

\begin{abstract}
We have investigated the pressure- and temperature-induced conformational changes associated with the low complexity domain of hnRNP A1, an RNA-binding protein able to phase separate in response to cellular stress. Solution NMR spectra of the hnRNP A1 low-complexity domain fused with protein-G B1 domain were collected from 1 to 2,500 bar and from $268 \mathrm{~K}$ to $290 \mathrm{~K}$. While the GB1 domain shows the typical pressure-induced and cold temperature-induced unfolding expected for small globular domains, the low-complexity domain of hnRNP A1 exhibits unusual pressure and temperature dependences. We observed that the low-complexity domain is pressure sensitive, undergoing a major conformational transition within the prescribed pressure range. Remarkably, this transition has the inverse temperature dependence of a typical folding-unfolding transition. Our results suggest the presence of a low-lying extended, and fully solvated state(s) of the low-complexity domain that may play a role in phase separation. This study highlights the exquisite sensitivity of solution NMR spectroscopy to observe subtle conformational changes and illustrates how pressure perturbation can be used to determine the properties of metastable conformational ensembles.
\end{abstract}

\section{Hosted file}

200831_hnRNPA1-Proteins.pdf available at https://authorea.com/users/355264/articles/478590thermodynamic-stability-of-hnrnp-a1-low-complexity-domain-revealed-by-high-pressure-nmr 